\title{
Review: exploring the potential of Nigella sativa for tooth mineralization and periodontitis treatment and its additive effect with doxycycline
}

Nurul Fitriatul Akmal Ismail ${ }^{1}$, Muhamad Ashraf Rostam ${ }^{2,5 *}$, Muhammad Fadhill Md Jais ${ }^{1}$, Mohd Affendi Mohd Shafri ${ }^{1}$, Ahmad Faisal Ismail ${ }^{3,5}$, Mohd Hafiz Arzmi 4,5

1 Department of Biomedical Sciences, Kulliyyah of Allied Health Sciences, International Islamic University Malaysia (IIUM), 25200 Kuantan, Pahang, Malaysia

2 Department of Nutritional Sciences, Kulliyyah of Allied Health Sciences, International Islamic University Malaysia (IIUM), 25200 Kuantan, Pahang, Malaysia

${ }^{3}$ Department of Paediatric Dentistry and Dental Public Health, Kulliyyah of Dentistry, International Islamic University Malaysia (IIUM), 25200 Kuantan, Pahang, Malaysia

${ }^{4}$ Department of Fundamental Dental and Medical Sciences, Kulliyyah of Dentistry, International Islamic University Malaysia (IIUM), 25200 Kuantan, Pahang, Malaysia

${ }^{5}$ Cluster for Cancer Research Initiative International Islamic University Malaysia (COCRII), 25200 Kuantan, Pahang, Malaysia

\begin{abstract}
Oral disease has been a worldwide concern as the incidence and cost of treatment continues to rise. Management of the disease is challenging as success is often influenced by an individual's lifestyle and diet. Nigella sativa ( $N$. sativa) or black seed is a medicinal plant that has received growing interest due to its effectiveness against various conditions including cardiovascular disease, diabetes, cancer, as well as infectious diseases caused by bacterial, viral, and fungal infections. This narrative review studies $N$. sativa that has also shown great potential in dental health attributed to its role in stimulating the process of remineralization. Apart from that, $N$. sativa promotes healing of oral tissues as it induces the differentiation potential of heterogeneous cell populations in periodontal ligament cells. The anti-osteoporotic, antioxidant and anti-inflammatory properties of $N$. sativa also improve periodontal healing, particularly by preventing further destruction of bone components. This article discusses the occurrence of periodontitis and the therapeutic role of $N$. sativa with emphasis on the regulation of genes, for instance, the alkaline phosphatase $(A L P)$ that is involved in tissue specialization and remineralization. It is speculated that the activity of $N$. sativa in remineralization can be enhanced by combination with other commonly used periodontitis antibiotics such as doxycycline. $N$. sativa is purported to induce periodontal tissue regeneration whilst minimizing toxicity. Thus, it may serve as an alternative or enhance the existing treatment for periodontitis when administered as a combined formulation.
\end{abstract}

Keywords: doxycycline, gene expression, mineralization, Nigella sativa, periodontitis

\section{Received:}

10 January 2022

Revised:

7 February 2022

Accepted:

7 February 2022

Published Online:

4 March 2022

How to cite this article:

Ismail, N. F. A., Rostam, M. A. .,

Md Jais, M. F., Mohd Shafri, M. A.

., Ismail, A. F., \& Arzmi, M. H.

Review: exploring the potential of Nigella sativa for tooth mineralization and periodontitis treatment and its additive effect with doxycycline. IIUM Journal of Orofacial and Health Sciences, 3(1), 136-146. https://doi.org/10.31436/ijoh s.v3i1.120

\section{Article DOI:}

10.31436/ijohs.v3i1.120

*Corresponding author

Address:

Department of Nutrition Sciences, Kulliyyah of Allied Health Sciences, International Islamic University Malaysia (IIUM), 25200 Kuantan, Pahang, Malaysia.

Telephone: +609-570 5309,

+6011-27059136

Email address:

ashrafrostam@iium.edu.my

\section{Introduction}

Periodontal disease and dental caries affect up to $50 \%$ of the worldwide population. The percentage of affected individuals are higher among adults and older group of people (Nazir, 2017). The economic impact, whether directly or indirectly, due to 
periodontal disease treatments, accounted for about 442 billion USD per year (Tonetti et al., 2017). These data portray the serious implication of periodontal disease not only to an individual's oral health but also financial status. The Malaysian healthcare system, through its dental services, spent approximately RM 2,800 per patient to treat periodontal diseases (Mohd-Dom et al., 2013). A study by Jin et al. (2016) stated that chronic periodontitis is among the global disease burden and affected almost $12 \%$ of people worldwide between the years 1990 to 2010 .

Periodontal disease or gum disease can be characterized into two conditions which include gingivitis and periodontitis. Gingivitis is the mildest form that causes the gum to be red, swollen and tend to bleed easily. This condition is often reversible as the main cause of gingivitis is poor oral hygiene (Shaw et al., 2016). Nevertheless, if gingivitis is left untreated, eventually periodontitis may develop. This occurs due to plaque accumulation reaching beyond the gum line. The presence of bacteria and its toxin initiates an inflammatory response, and eventually, chronic inflammation in the gingiva will slowly destroy the gum tissue and teeth, gingival, periodontal ligament, cementum, and the alveolar bone. As the gingival tissue is inflamed, the tissue itself loses its attachment to the tooth surface, creating a periodontal pocket (Arigbede et al., 2012).

Periodontal disease can be classified into four categories. Firstly, aggressive periodontitis where there is a rapid attachment loss between gingiva and tooth as well as alveolar bone destruction. Secondly, chronic periodontitis which involves inflammation of the periodontium, and this causes the progressive loss of attachment and bone destruction. The main difference between aggressive and chronic periodontitis is the onset. Aggressive periodontitis tends to occur earlier as compared to chronic periodontitis. Wiebe and Putnins (2000) mentioned that the three criteria that govern the categorization are the depth of the pocket, the clinical attachment loss and the number of teeth affected whether more or less than $30 \%$. The third type of periodontitis may be the result of a systemic disease including respiratory disease, heart disease and diabetes. The effects are suggested to occur due to the infection of bacteria in systemic circulation and cytokine storm released from the extensive inflammatory response. The fourth type is necrotizing periodontitis which affects mostly immunocompromised and malnourished individuals. This condition is attributed to necrosis of the periodontium, gingival tissue, periodontal ligament, and alveolar bone (Wiebe \& Putnins, 2000).

Currently, gingivitis can be prevented and treated by advocation of proper oral hygiene such as frequent tooth brushing. However, for periodontitis, a comprehensive periodontal treatment is required, which may include a non-surgical procedure called scaling and root planing (SRP). This procedure is performed by a dentist to scale all the plaque, bacterial toxins and tartar deposition on the tooth and root surfaces followed by root planing. Root planing smoothens out the surfaces and prevents any re-adherence of plaque or tartar, allowing a condition that induces the healing processes and reattachment of the gum to the tooth (Smiley et al., 2015). SRP is also referred to as deep cleaning, a gold standard in treating periodontitis in which the procedure is commonly coupled with treatment using tetracycline antibiotics, specifically the doxycycline hyclate (Tomasi \& Wennström, 2004). Although the treatment is a common practice, the procedure has been reported to be technically challenging and may cause recurrence. The problem with current treatment is that SRP itself induces pain to the patients and may cause patients to retract from getting treatment. The second problem is the presence of bacterial biofilm at the site of treatment. Bacterial biofilm which is embedded in layers has optimal defence against antibiotic effect when compared to the planktonic bacteria (Dincer et al., 2020). Due to the resistant properties, higher doses of antimicrobial as high as 10 to 1000 times will be required than normal to kill it (Gebreyohannes et al., 2019). However, 
the administration of drugs at high dosage can cause local toxicity affecting the surrounding area (Shaddox \& Walker, 2010). This, in turn, hinders the remineralization of tooth and dentin structures (Holmes \& Charles, 2009; Ioannou et al., 2010).

Continuous studies were conducted to improve the treatment for periodontitis (Alshareef et al., 2020; Shaddox \& Walker, 2010). It is postulated that an effective therapeutic strategy is to provide a comprehensive treatment that could deliver antimicrobial protection, reduce inflammation, and stimulate remineralization of the alveolar bone. The use of phytomedicine has gained a lot of interest particularly in targeting a wide range disease with more accessible, costeffective, and fewer side effects in comparison to the synthetic medicines (Yimer et al., 2019). Among medicinal plants, $N$. sativa has been considered one of the highly valued nutrient-rich plants in history around the world. A lot of studies are progressively conducted to validate its traditionally claimed benefits and use (Ramadan, 2007).

Research on the use of traditional medicines opens new boundaries for the alternative approach in the management of oral diseases (Cruz et al., 2017). The World Health Organization (WHO) defines traditional medicine as any sum of total knowledge, skill and practices which comes from a particular culture through theories, beliefs, and experiences in health-related conditions. Traditional medicine is identical to modern medicine in that it aims to provide maintenance of health which also paves way for improvement of life quality (Chaudhary \& Singh, 2011). In fact, traditional medicine has long existed before modern medicine hundreds of years earlier and has been proved to contribute fruitful finding to modern medicine such as the emergence of the first pharmacologically active compound morphine in the year 1805 (Yuan et al., 2016). N. sativa is a medicinal plant that has been studied for its antimicrobial and antiinflammatory properties (Ahmad et al., 2013; Al-Ghamdi, 2001; Forouzanfar et al., 2014). In this review, the therapeutic role of
$N$. sativa as a comprehensive bioactive natural product that can be an alternative treatment modality for periodontitis is discussed, particularly via promoting remineralization of the tooth and alveolar bone. A detailed emphasis is given on the effects of $N$. sativa towards genes involved in remineralization.

\section{Methodology}

This article adopts the narrative review approach in which relevant articles written in English were retrieved from Scopus, Science Direct and Google Scholar database. Articles and books from the year 1991 to 2020 were selected to include fundamental knowledge on teeth and its development as well as recent studies related to $N$. sativa, mineralization and its impact towards periodontitis. In the light of genetic impact of $N$. sativa on alkaline phosphatase expression, we included keyword search of "Nigella sativa and its constituents' therapeutic properties", "mineralization", "alveolar bone remodelling", "periodontal ligament gene expression", "ALP gene expression" and "N. sativa genetic impact". Six themes were covered under the following subtopics: (1) Teeth mineralization, demineralization, and remineralization, (2) Periodontal tissue, (3) Gene expression of the periodontal ligament, (4) N. sativa and its therapeutic properties on dental health, (5) N. sativa effects on teeth mineralization and $A L P$ expression, (6) $N$. sativa additive effect. This review also covers the possible use of $N$. sativa coupled with antibiotics to enhance its effect in treatment.

\section{Teeth \\ demineralization, mineralization, remineralization \\ and}

Human teeth are structurally formed by three layers which include the enamel, pulpdentin complex, and cementum (Figure 1). Enamel is the outermost whitish calcified layer that makes up the crown structure and teeth root. Pulp-dentin complex is present in the inner layer consisting of dentin as the immediate layer after enamel and pulp having all the capillaries supplying blood 
and nerve to the teeth. Cementum covers the root surface to maintain dental and periodontal attachment (Boskey, 2007). For dentin and cementum, almost $90 \%$ of these structures are made up from collagen, specifically from collagen type I. On the other hand, nearly $90 \%$ of the enamel structure is composed of non-collagenous protein (Margolis et al., 2014).

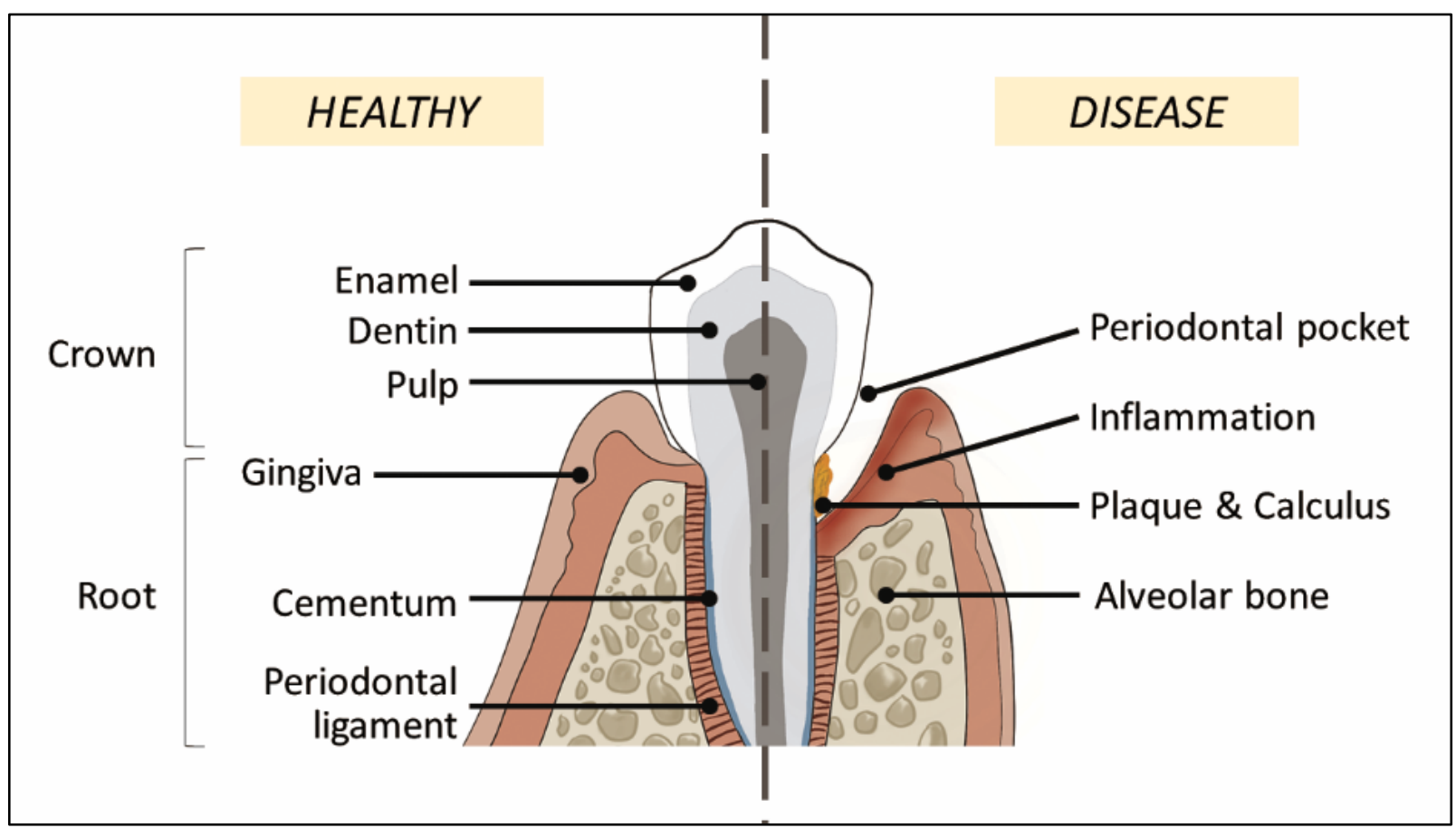

According to Caruso et al. (2016), mineralization starts at the dentin structure through a process known as dentinogenesis. This process is further subdivided into three distinct stages dependent on the site of mineralization. The first stage is vesicle derived mineralization that occurs in the mantle or the outer layer of dentin. Within odontoblastic processes, matrix vesicles (MVs) are vesicular bodies found in dentin and are present in the extracellular environment during the mantel dentine mineralization process (Garcés-Ortíz, 2013). MVs are formed via budding events of odontoblasts that reside in the basement membrane. The vesicles produced would then provide initial mineralization components, including enzymes required for dentin (Golub, 2009). The second stage is the extracellular matrix (ECM) moleculederived mineralization. This process appears when odontoblasts secrete type I collagen and proteoglycans, particularly chondroitin sulphate which functions to attract calcium ions and acts as a transporter for mineral from cells into the ECM. These components make up the matrix of dentin and induce differentiation of pre-dentin to dentin. Some non-collagenous proteins (NCPs) are also being secreted influencing the nucleation and also inhibition of mineralization. Finally, the third stage is blood-serum-derived mineralization where there is a passive deposition of proteoglycans, lipid and ECM secreted from odontoblasts or from the dentinal lymph. These depositions give rise to the formation of a thin amorphous matrix in the peritubular dentin (Goldberg et al., 2011). In the matrix-derived mineralization, type I collagen is the main component of dentin being incorporated equivalent to more than half of mineral contents while the are some parts of NCP present acting as a promoter or an inhibitor. These proteins inhibit immature mineralization of dentin until proper orientation and arrangement of collagen fibril are achieved (Van Der Rest \& Garrone, 1991). 
After the mineralization of dentin, the enamel structure undergoes a similar process by the action of ameloblasts cells which shares a common origin with odontoblasts from mesenchymal stem cells (Moradian-Oldak, 2012). These cells secrete enamel matrix protein and proteinases, which contribute to the immediate mineralization of enamel. Enamel crystal ribbon is initially formed within the mineralized dentin surface along with the secreted enamel matrix proteins that grow away outwards from the dentin. Consequently, full-scale enamel thickness is produced, causing ameloblasts to become protein-resorbing cells to remove enamel matrix proteins. Due to the removal, the structure is compensated by minerals and water making up the structure with approximately $95 \%$ mineral contents. Further calcification process of teeth occurs as calcium is transferred from the blood into the enamel matrix where they are deposited (Abou Neel et al., 2016).

Under normal circumstances, demineralization of the teeth occurs all the time, particularly when subjected to acidic contents from the diet as well as from normal oral microbiota (Abou Neel et al., 2016). Demineralization of the tooth indicates minerals wearing off from the enamel and dentin, which can cause the teeth to become porous. Nevertheless, the number of minerals being washed away are small and do not affect the deeper hard tissues. Saliva involves in the mechanism that the acid produced from diets or normal flora in the oral cavity. However, periodontitis, an inflammation event affecting the periodontium of the tooth, largely promotes demineralization of the teeth (Gasner \& Schure, 2020). Untreated and prolonged gingivitis leads to the extensive growth of bacterial biofilm deep beyond the gingival margin. This condition causes the deepening of the periodontal pocket (Figure 1). The condition worsens following the destruction of the periodontium (consisting of the periodontal ligament, cementum, gingiva, and alveolar bone) whereby demineralization that extends up to the alveolar bone, further weakens tooth attachment and ultimately causing tooth loss (Larsen \& Fieehn, 2017). Remineralization can be promoted by keeping away the bacterial plaque from depositing in the teeth through proper brushing and flossing the teeth. Another way is to use fluoride contained toothpaste as it strengthens and induce remineralization. Vitamin D and natural products may also help in promoting remineralization (Philip, 2019). Factors that promote remineralization includes the timedependent exposure of tooth surface to the calcium, phosphate and fluoride brought by the saliva (Garcia-Godoy \& Hicks, 2008).

\section{Periodontal tissue}

Periodontal tissue is located between the cementum and the alveolar bone, as depicted in Figure 1. It is characterized by the dense fibrous connective tissue that acts as a support medium and attachment for the teeth to its socket. The major component of periodontal tissue essential for normal teeth function is the periodontal ligament (PDL) which contains a heterogeneous cell population that are fibroblastic in nature as well as cells that contribute to mineralized tissue formation (Dean, 2017; Maeda et al., 2011; Marchesan et al., 2011).

This remodelling capacity allows it to differentiate into specialized tissues which are bone, cementum, and connective tissue fibres. Meanwhile, the undifferentiated cells present around the blood vessels, namely ectomesenchymal cells, have the capability to differentiate into cementoblasts, osteoblasts and fibroblasts (Caetano-Lopes et al., 2007).

The PDL functions as a supportive structure that strengthens the teeth attached to the alveolar bone socket (Dean, 2017). The principal fibres of the periodontal ligament are the key element that serves as a bridge that unites the root cementum with the bone by strong fibrous connection (Matsuo \& Takahashi, 2002). Besides, the periodontal ligament, particularly the principal fibres, also acts as an attachment medium and shock-absorber that can withstand heavy 
occlusal force during mastication (Li et al., 2018). In addition, blood-origin macrophage also supplies the area with multinucleated cells which are osteoclasts and odontoclasts. These two cells are involved in the process of bone and tooth resorption (Dean, 2017).

Other functions of the PDL are sensory and nutritive. Nerve ends are deposited inside the periodontal ligament, providing receptors for pain and pressure (Dean, 2017). The periodontal ligament also possesses well-vascularization which arises from the dental arteries. This vascularization provides nutrient for the cell surrounding vitality (Dean, 2017).

\section{Gene expression of the periodontal ligament}

According to Choi et al. (2011), the gene expression involved in osteogenic events of PDL follows a temporal sequence beginning from the proliferation stage, subsequently the bone matrix formation and maturation stage, and finally the mineralization stage. During the proliferation stage, $c-m y c$, a cell growth-regulated gene expressed in PDL encodes proteins that induce proliferation by functioning as transactivation factors. After cell proliferation is downregulated, alkaline phosphatase $(A L P)$ mRNA expression is elevated to enter the cell differentiation stage. $A L P$ is one of the biomarkers that influence PDL cell differentiation into osteoblastic cells (Choi et al., 2011). As the bone matrix continues to develop, $A L P$ expression elevates, thus making the protein as one of the markers for osteoblastic cells.

Human $A L P$ is categorized into four subtypes which are tissue nonspecific, intestinal, placenta and germ cells. Tissue nonspecific ALP (TNAP) is responsible for bone and teeth mineralization (Millán, 2013). TNAP is mainly expressed by chondrocytes, osteoblasts, and odontoblasts (Chaudhary et al., 2016; Hoylaerts et al., 2015). TNAP is concentrated in the vesicles which bud off from those cells to supply through vesicle-derived mineralization. During the mineralization process, TNAP activity catalyses the conversion of PPi into Pi. PPi is an inhibitor of hydroxyapatite crystal formation. Meanwhile, $\mathrm{Pi}$ is needed to form hydroxyapatite crystal (Orimo, 2010). $A L P$ gene is located on chromosome $2 \mathrm{q} 37.1$ (Lee et al., 2007). Inhibition of $A L P$ gene expression may halt the mineralization process leading to hypomineralization, thus highlighting its crucial role (Orimo \& Shimada, 2008).

During PDL differentiation, the $B M P$ gene was also expressed, particularly $B M P-2$ and BMP-4. BMP-2 and -4 showed similar expression patterns to $A L P$ (Choi et al., 2011). $B M P$ is important as a chemoattractant for differentiation of undifferentiated mesenchymal stem cells into areas of bone loss, thus increasing the bone matrix protein. Besides being expressed in the PDL fibroblasts, gingival fibroblasts also express $B M P$ and $A L P$ (Rahman et al., 2015).

The active expression of $A L P$ was followed by the expression of osteocalcin (OC). OC is a protein that is routinely used as a serum marker for osteoblastic bone formation and currently suggested to demonstrate hormonal roles that extend to involve regulation of whole-body metabolism, cognition, and reproduction (Zoch et al., 2016). The increase in $O C$ gene expression in PDL cells was shown to correlate with the formation of bone nodules. At the terminal differentiation of osteoblastic cells, c-fos expression was prominent (Choi et al., 2011). c-fos is a cell growth-regulated transcription factor that has a specific association with bone tissue (Yamaguchi et al., 2002).

Other than that, in the events of infection or orthodontic tooth movement due to orthodontic force, interleukin-1 (IL-1) and interleukin-8 (IL-8) are expressed in PDL, which invoke an early inflammatory response and osteoclastic bone resorption (Lee et al., 2013). IL-8 plays a key role in neutrophils activation and recruitment whereas IL-1ß is an important bone resorption cell mediator (Tuncer et al., 2005). Following traumatic condition, necrotic cells are formed, and the nearest 
healthy ligament cell will aid in repair. However, excessive chemokine response may develop chronic hyperinflammatory lesion leading to periodontal ligament and alveolar bone loss (Huang et al., 1999; Nibali et al., 2013). It is evident that IL-1 is essential in the pathogenesis of periodontitis. Therefore, the genes encoding IL-1 production had been regarded as a potential predictor of periodontal disease progression (Grigoriadou et al., 2010).

\section{Nigella sativa ( $N$. sativa) and its therapeutic properties on dental health}

$N$. sativa or commonly known as black seed or Habbatus sauda' is a medicinal seed that is known for its therapeutic effects. $N$. sativa seed and its extracted oil are used as a therapy due to its wide properties including antimicrobial and anti-inflammatory. The main active compound of $N$. sativa is thymoquinone $(30-40 \%)$ which is proposed to be responsible for the antimicrobial and anti-inflammatory properties (Ahmad et al., 2013). Thymoquinone (TQ) has been shown to possess antimicrobial effects demonstrated by the growth inhibition of bacteria such as Staphylococcus aureus ( $S$. aureus) when treated with the active compound. TQ has been demonstrated to be more effective against gram-negative bacteria and that with wide range of antibiotic resistance (Ahmad et al., 2013). Anti-inflammatory effects of TQ are attributed to its ability to inhibit key inflammatory cytokines such as IL-1 and nuclear factor $\kappa \mathrm{B}$. Besides $\mathrm{TQ}$, other bioactive constituents of $N$. sativa such as dithymoquinone, thymol, and thymohydroquinone are also being studied for their therapeutic properties (Gholamnezhad et al., 2016).

In relation to oral care, TQ offers a promising therapeutic candidate due to its ability to reduce dental caries and plaque index of teeth (Al-Attas et al., 2016; Angius et al., 2015). TQ is proved to be effective as an inhibitory agent against attachment and colonization of dental microbes such including Streptococcus mitis (S. mitis) and
Streptococcus mutans (S. mutans) (Harzallah et al., 2011). Besides influencing bacterial colonization, TQ also exerts both antioxidant and anti-inflammatory effects. Periodontitis can be caused by oxidative stress in which reactive oxygen species (ROS) increase beyond the tolerable limit of antioxidant capabilities. Diminished antioxidant scavenging activities lead to periodontal tissue destruction through lipid peroxidation. In the case of Porphyromonas gingivalis ( $P$. gingivalis) induced inflammation through its lipopolysaccharide (LPS), which causes the excessive formation of mitochondrial ROS. TQ, with its antioxidant properties, is suggested to induce ROS scavenging activity, thus reducing the further breakdown of periodontal tissues (Bullon et al., 2014). Thus, this impedes the action of ROS that disrupts with the osteoclastic activity. TQ has been shown to reduce alveolar bone loss, infiltration of inflammatory cells and osteoclasts activities while improving osteoblastic differentiation on periodontium (Al-Ghamdi, 2001). Therefore, the coupled effect of the antimicrobial and anti-oxidative property of TQ is suggested to be a suitable candidate in the formulation of treatments in the management of periodontitis.

\section{$N$ sativa effects on teeth mineralization and $A L P$ expression}

$N$. sativa can induce anti-osteoporotic conditions due to its antioxidant and antiinflammatory properties that prevent the destruction of bone components (Rukshar \& Neha, 2013). These properties are beneficial in treating periodontitis. $N$. sativa has been reported to induce the expression of $A L P$ which is linked to the mineralized-tissueforming cell differentiation as well as increasing bone nodule formation (Wirries et al., 2013). Other genes such as $O C$, osteopontin $(O P N)$ and $B M P$ gene are also stimulated by $N$. sativa specifically by its active component the thymoquinone (Islam et al.; 2016Wirries et al., 2013). BMP genes, particularly $B M P-2$, increases its expression through the ERK pathway, which is known to involve in bone formation and osteoblast 
differentiation (Jun et al., 2010; Su et al., 2010).

Alveolar bone loss is a key feature of periodontitis progression, and this condition is associated with enhanced osteoclast formation. Periodontitis induces osteoclast formation by increasing the differentiation of quiescent osteoclast precursors into osteoclasts (Lee et al., 2015). Thummuri et al. (2015) highlighted that TQ could reduce osteoclastogenic activity exerted by receptor activator of nuclear factor kappa B ligand (RANKL) through inhibition of MAPK and NF-kB signalling pathway. NF-kB and MAPK are involved in osteoclast differentiation alongside with JUNK and p38. Through activation of the MAPK pathway, $c$-fos is induced, thus increasing the osteoclastogenesis, which leads to bone resorption (Boyle et al., 2003; Lee et al., 2009). Meanwhile, inhibition of TQ halts both the activation of MAPK and p38, leading to decreased cellular differentiation of osteoclasts, in turn reducing its downstream effects.

The destructive effects of periodontitis are also incited by subgingival plaque microflora and LPS derived from pathogens (Hienz et al., 2015). TQ could also prevent osteoclastogenic activity by LPS through the MAPK, NF-kB and TLR signalling pathway (Thummuri et al., 2015). This is complementary to the antimicrobial properties of TQ, which is beneficial to the suppression of bacterial biofilm formation.

\section{$N$. sativa additive effect}

$N$. sativa has been reported to possess additive effect in eliminating microbial when combined with doxycycline (Aljabre et al., 2015; Forouzanfar et al., 2014; Halawani, 2009). Doxycycline is an antibiotic in tetracycline class that has frequently been used for periodontitis treatment (Garret et al., 1999; Kopytynska-Kasperczyk et al., 2015). In fact, doxycycline is semi synthetic and more active compared to other antibiotic in the tetracycline class. Doxycycline is effective in eliminating many bacteria species includes enterococci,
Streptococcus pyogenes, anaerobic, various Nocardia spp. and $S$. aureus which is originally resistant to tetracycline (Kogawa \& Salgado, 2012). Doxycycline is bacteriostatic, exerting antibacterial action by inhibiting bacterial growth and prevent the bacteria from reproducing (Loree \& Lappin, 2019). Doxycycline binds to the 30S ribosomal subunit in protein translation process to stop amino acid from being linked and inhibit the bacterial protein synthesis (Kogawa \& Salgado, 2012; Raval et al., 2018). Besides, it has anti-collagenase properties that can help to protect and maintain the collagen composition on the site of treatment (Raval et al., 2018) while at the same time eradicating the pathogenic bacteria. On the downside, doxycycline must be administered at proper dose as gastrointestinal effect will arise from the usage of the antibiotic such as stomach irritation, ulceration (Raval et al., 2018) and phototoxic reaction (Skidmore et al., 2003). Hence, addition of any compound on top of doxycycline that can buffer the toxic effect can be considered to ensure an effective treatment.

\section{Conclusion}

Traditional medicine had used medicinal herbs as therapeutic agents to cure oral diseases. It has been suggested that $N$. sativa or black seed have great benefits in improving oral health. Evidence of a coupled effect (antimicrobial and antioxidant) of TQ towards the main pathogenicity of periodontitis further highlights its potential as an alternative treatment modality. Further research is essential to study the optimal dosage of $N$. sativa that is therapeutically effective as well as analysis on the toxicological profile to ensure the safety. Several genes, especially the $A L P$ gene, can be a suitable candidate as the downstream target.

$N$. sativa coupled with tetracycline has an additive effect against pathogenic bacteria. In this light, a possible therapeutic approach is that $N$. sativa can be combined with doxycycline, a type of tetracycline antibiotic to serve as antimicrobial, remineralization 
and regeneration agent for remediating cell structures damaged by periodontitis. Nevertheless, the correct formulation is needed to achieve stability for the formulation. Doxycycline can be effective, but without proper dosage quantification and without additional compounds to enhance its efficacy, disease recurrence is inevitable.

\section{Conflict of interest}

The authors declare no conflict of interest and that the review was conducted in the absence of any financial relationships that could be construed as a potential conflict of interest.

\section{Acknowledgement}

This study was supported by the International Islamic University Malaysia (IIUM) Research Initiative Grant Scheme (RIGS17-117-0692) and Fundemental Research Grant Scheme (FRGS16-0490548). We also thank the Department of Paediatric Dentistry and Dental Public Health and Department of Fundamental Dental and Medical Sciences of Kulliyyah of Dentisty, IIUM in support for the conduct of this review

\section{References}

Abou Neel, E.A., Aljabo, A., Strange, A., Ibrahim, S., Coathup, M., Young, A.M. et al. (2016). Demineralization-remineralization dynamics in teeth and bone. International Journal of Nanomedicine, 11, 4743.

Ahmad, A., Husain, A., Mujeeb, M., Khan, S.A., Najmi, A.K., Siddique, N.A. et al. (2013). A review on therapeutic potential of Nigella sativa: A miracle herb. Asian Pacific Journal of Tropical Biomedicine, 3(5), 337-352.

Al-Attas, S.A., Fat'heya, M.Z., Turkistany, S.A. (2016). Nigella sativa and its active constituent thymoquinone in oral health. Saudi Medical Journal, 37(3), 235.

Al-Ghamdi, M.S. (2001). The anti-inflammatory, analgesic and antipyretic activity of Nigella sativa. Journal of Ethnopharmacology, 76(1), 45-48.

Aljabre, S.H., Alakloby, O.M., Randhawa, M.A. (2015). Dermatological effects of Nigella sativa. Journal of Dermatology and Dermatologic Surgery, 19(2), 9298.

Alshareef, A., Attia, A., Almalki, M., Alsharif, F., Melibari, A., Mirdad, B. et al. (2020). Effectiveness of probiotic lozenges in periodontal management of chronic periodontitis patients: clinical and immunological study. European Journal of Dentistry, 14(2), 281.

Angius, F., Madeddu, M.A., Pompei, R. (2015). Nutritionally variant streptococci interfere with Streptococcus mutans adhesion properties and biofilm formation. New Microbiologica, 38(2), 25966.

Arigbede, A.O., Babatope, B.O., Bamidele, M.K. (2012). Periodontitis and systemic diseases: a literature review. Journal of Indian Society of Periodontology, 16(4), 487.

Boskey, A.L. (2007). Mineralization of bones and teeth. Elements, 3(6), 385-391.

Boyle, W.J., Simonet, W.S., Lacey, D.L. (2003). Osteoclast differentiation and activation. Nature, 423(6937), 337-342.

Bullon, P., Newman, H.N., Battino, M. (2014). Obesity, diabetes mellitus, atherosclerosis and chronic periodontitis: a shared pathology via oxidative stress and mitochondrial dysfunction?. Periodontology 2000, 64(1), 139-153.

Caetano-Lopes, J., Canhao, H., Eurico Fonseca, J. (2007). Osteoblasts and bone formation. Acta Reumatológica Portuguesa, 32(2).

Caruso, S., Bernardi, S., Pasini, M., Giuca, M.R., Docimo, R., Continenza, M.A. et al. (2016). The process of mineralisation in the development of human tooth. European Journal of Paediatric Dentistry, 17(4), 322-326.

Chaudhary, A., Singh, N. (2011). Contribution of World Health Organization in the global acceptance of Ayurveda. Journal of Ayurveda and Integrative Medicine, 2(4), 179.

Chaudhary, S.C., Kuzynski, M., Bottini, M., Beniash, E., Dokland, T., Mobley, C.G. et al. (2016). Phosphate induces formation of matrix vesicles during odontoblast-initiated mineralization in vitro. Matrix Biology, 52, 284-300.

Choi, M.H., Noh, W.C., Park, J.W., Lee, J.M., Suh, J.Y. (2011). Gene expression pattern during osteogenic differentiation of human periodontal ligament cells in vitro. Journal of Periodontal and Implant Science, 41(4), 167-175.

Cruz Martinez, C., Diaz Gomez, M., Oh, M.S. (2017). Use of traditional herbal medicine as an alternative in dental treatment in Mexican dentistry: a review. Pharmaceutical Biology, 55(1), 1992-1998.

Dean, R. (2017). The periodontal ligament: development, anatomy and function. Oral Health Dental Management, 16(6).

Dincer, S., Uslu, F.M., Delik, A. (2020). Antibiotic Resistance in Biofilm. In: Dincer S, Ozdenefe MS, Arkut A, Bacterial Biofilms. London: IntechOpen, pp. 135-148.

Forouzanfar, F., Bazzaz, B.S.F., Hosseinzadeh, H. (2014). Black cumin (Nigella sativa) and its constituent (thymoquinone): a review on antimicrobial effects. Iranian Journal of Basic Medical Sciences, 17(12), 929.

Garcés-Ortíz, M., Ledesma-Montes, C., Reyes-Gasga, J. (2013). Presence of matrix vesicles in the body of odontoblasts and in the inner third of dentinal tissue: A scanning electron microscopyc study. Medicina Oral, Patologia Oral y Cirugia Bucal, 18(3), e537. 
García-Godoy, F., Hicks, M.J. (2008). Maintaining the integrity of the enamel surface: the role of dental biofilm, saliva and preventive agents in enamel demineralization and remineralization. The Journal of the American Dental Association, 139, 25S-34S.

Garrett, S., Johnson, L., Drisko, C.H., Adams, D.F., Bandt, C., Beiswanger, B. et al. (1999). Two multi-center studies evaluating locally delivered doxycycline hyclate, placebo control, oral hygiene, and scaling and root planing in the treatment of periodontitis. Journal of Periodontology, 70(5), 490-503.

Gasner, N.S, Schure, R.S. (2020). Periodontal Disease. In: StatPearls. Treasure Island FL: StatPearls Publishing LLC.

Gebreyohannes, G., Nyerere, A., Bii, C., Sbhatu, D.B. (2019). Challenges of intervention, treatment, and antibiotic resistance of biofilm-forming microorganisms. Heliyon, 5(8), e02192.

Gholamnezhad, Z., Havakhah, S., Boskabady, M.H. (2016). Preclinical and clinical effects of Nigella sativa and its constituent, thymoquinone: A review. Journal of Ethnopharmacology, 190, 372-386.

Goldberg, M., Kulkarni, A.B., Young, M., Boskey, A. (2011). Dentin: structure, composition and mineralization: the role of dentin ECM in dentin formation and mineralization. Frontiers in Bioscience (Elite edition), 3, 711.

Golub, E.E. (2009). Role of matrix vesicles in biomineralization. Biochimica et Biophysica Acta (BBA)-General Subjects, 1790(12), 1592-1598.

Grigoriadou, M.E., Koutayas, S.O., Madianos, P.N., Strub, J.R. (2010). Interleukin-1 as a genetic marker for periodontitis: Review of the literature. Quintessence International, 41(6), 517-25.

Halawani, E. (2009). Antibacterial activity of thymoquinone and thymohydroquinone of Nigella sativa L. and their interaction with some antibiotics. Advances in Biological Research, 3(5-6), 148-152.

Harzallah, H.J., Kouidhi, B., Flamini, G., Bakhrouf, A., Mahjoub, T. (2011). Chemical composition, antimicrobial potential against cariogenic bacteria and cytotoxic activity of Tunisian Nigella sativa essential oil and thymoquinone. Food Chemistry, 129(4), 1469-1474.

Hienz, S.A., Paliwal, S., Ivanovski, S. (2015). Mechanisms of bone resorption in periodontitis. Journal of Immunology Research, 2015.

Holmes, N.E., Charles, P.G. (2009). Safety and efficacy review of doxycycline. Clinical Medicine. Therapeutics, 1, 471-482.

Hoylaerts, M.F., Kiffer-Moreira, T., Sheen, C., Narisawa, S., Millán, J.L. (2015). Functional significance of calcium binding to tissue-nonspecific alkaline phosphatase. PloS one, 10(3), e0119874.

Huang, G. T. J., Potente, A. P., Kim, J. W., Chugal, N., \& Zhang, X. (1999). Increased interleukin-8 expression in inflamed human dental pulps. Oral Surgery, Oral Medicine, Oral Pathology, Oral Radiology, and Endodontology, 88(2), 214-220.

Ioannou, I., Dimitriadis, N., Papadimitriou, K., Vouros, I., Sakellari, D., Konstantinidis, A. (2010). The effect of locally delivered doxycycline in the treatment of chronic periodontitis. A clinical and microbiological cohort study. Journal of Oral and Maxillofacial Research, 1(4), e1.

Islam, M.T., Sultana, N., Riaz, T.A., Ferdous, J., Guha, B., Mohagon, S. et al. (2016). Thymoquinone is knocking at the door of clinical trial. International Archives of Medicine, 9(122), 1-25.

Jin, L.J., Lamster, I.B., Greenspan, J.S., Pitts, N.B., Scully, C., Warnakulasuriya, S. (2016). Global burden of oral diseases: emerging concepts, management and interplay with systemic health. Oral Diseases, 22(7), 609-619.

Jun, J.H., Yoon, W.J., Seo, S.B., Woo, K.M., Kim, G.S., Ryoo, H.M. et al. (2010). BMP2-activated Erk/MAP kinase stabilizes Runx2 by increasing p300 levels and histone acetyltransferase activity. Journal of Biological Chemistry, 285(47), 36410-36419.

Kogawa, A.C., Salgado, H.R.N. (2012). Doxycycline hyclate: a review of properties, applications and analytical methods. International Journal of Life Science and Pharmaceutical Research, ISSN, 22500480 .

Kopytynska-Kasperczyk, A., Dobrzynski, P., Pastusiak, M., Jarzabek, B., Prochwicz, W. (2015). Local delivery system of doxycycline hyclate based on $\epsilon$ caprolactone copolymers for periodontitis treatment. International Journal of Pharmaceutics, 491(1-2), 335-344.

Larsen, T., Fiehn, N.E. (2017). Dental biofilm infections-an update. Apmis, 125(4), 376-384.

Lee, D.E., Kim, J.H., Choi, S.H., Cha, J.H., Bak, E.J., Yoo, Y.J. (2015). Periodontitis mainly increases osteoclast formation via enhancing the differentiation of quiescent osteoclast precursors into osteoclasts. Journal of Periodontal Research, 50(2), 256-264.

Lee, Y.H., Nahm, D.S., Jung, Y.K., Choi, J.Y., Kim, S.G., Cho, M. et al. (2007). Differential gene expression of periodontal ligament cells after loading of static compressive force. Journal of Periodontology, 78(3), 446-452.

Lee, H.S., Lee, J., Kim, S.O., Song, J.S., Lee, J.H., Lee, S.I. et al. (2013). Comparative gene-expression analysis of the dental follicle and periodontal ligament in humans. PloS one, 8(12), e84201.

Lee, M.S., Kim, H.S., Yeon, J.T., Choi, S.W., Chun, C.H., Kwak, H.B. et al. (2009). GM-CSF regulates fusion of mononuclear osteoclasts into bone-resorbing osteoclasts by activating the Ras/ERK pathway. The Journal of Immunology, 183(5), 3390-3399.

Li, Y., Jacox, L.A., Little, S.H., Ko, C.C. (2018). Orthodontic tooth movement: The biology and clinical implications. The Kaohsiung Journal of Medical Sciences, 34(4), 207-214.

Loree, J., Lappin, S.L. (2019). Bacteriostatic Antibiotics. In: StatPearls. Treasure Island (FL): StatPearls Publishing LLC.

Maeda, H., Wada, N., Fujii, S., Tomokiyo, A., Akamine, A. (2011). Periodontal ligament stem cells. in Stem Cells. InTech, Croatia, 619-636.

Marchesan, J.T., Scanlon, C.S., Soehren, S., Matsuo, M., Kapila, Y.L. (2011). Implications of cultured periodontal ligament cells for the clinical and experimental setting: a review. Archives of Oral Biology, 56(10), 933-943.

Margolis, H.C., Kwak, S.Y., Yamazaki, H. (2014). Role of mineralization inhibitors in the regulation of hard tissue biomineralization: relevance to initial 
enamel formation and maturation. Frontiers in Physiology, 5, 339.

Matsuo, M., Takahashi, K. (2002). Scanning electron microscopic observation of microvasculature in periodontium. Microscopy Research and Technique, 56(1), 3-14.

Millán, J.L. (2013). The role of phosphatases in the initiation of skeletal mineralization. Calcified Tissue International, 93(4), 299-306.

Mohd-Dom, T.N., Abdul-Muttalib, K., Ayob, R., Lan, Y.S., Mohd-Asadi, A.S., Abdul-Manaf, M.R. et al. (2013). Periodontal status and provision of periodontal services in Malaysia: trends and way forward. Journal of Public Health Medicine, 13(2), 38-47.

Moradian-Oldak, J. (2012). Protein-mediated enamel mineralization. Frontiers in Bioscience: A Journal and Virtual Library, 17, 1996.

Nazir, M.A. (2017). Prevalence of periodontal disease, its association with systemic diseases and prevention. International Journal of Health Sciences, 11(2), 72.

Nibali, L., Fedele, S., Donos, N., \& D’Aiuto, F. (2013). The role of interleukin-6 in oral diseases. Dimensions of Dental Hygiene, 11(1), 28-34.

Orimo, H. (2010). The mechanism of mineralization and the role of alkaline phosphatase in health and disease. Journal of Nippon Medical School, 77(1), 412.

Orimo, H., Shimada, T. (2008). The role of tissuenonspecific alkaline phosphatase in the phosphateinduced activation of alkaline phosphatase and mineralization in SaOS-2 human osteoblast-like cells. Molecular and Cellular Biochemistry, 315(12), 51-60.

Philip, N. (2019). State of the art enamel remineralization systems: the next frontier in caries management. Caries Research, 53(3), 284295.

Rahman, M.S., Akhtar, N., Jamil, H.M., Banik, R.S., Asaduzzaman, S.M. (2015). TGF- $\beta$ /BMP signaling and other molecular events: regulation of osteoblastogenesis and bone formation. Bone Research, 3, 15005.

Ramadan, M.F. (2007). Nutritional value, functional properties and nutraceutical applications of black cumin (Nigella sativa L.): an overview. International Journal of Food Science and Technology, 42(10), 1208-1218.

Raval, J.P., Chejara, D.R., Ranch, K., Joshi, P. (2018). Development of injectable in situ gelling systems of doxycycline hyclate for controlled drug delivery system. In Applications of Nanocomposite Materials in Drug Delivery, 149-162. Woodhead Publishing.

Van Der Rest, M., Garrone, R. (1991). Collagen family of proteins. The FASEB Journal, 5(13), 2814-2823.

Rukshar, A., Neha, G.B. (2013). Effects of Nigella sativa against osteoporosis. International Journal of Pure and Applied Bioscience, 1(2), 6-14.

Shaddox, L.M., Walker, C.B. (2010). Treating chronic periodontitis: current status, challenges, and future directions. Clinical, Cosmetic and Investigational Dentistry, 2, 79.

Shaw, L., Harjunmaa, U., Doyle, R., Mulewa, S., Charlie, D., Maleta, K. et al. (2016). Distinguishing the signals of gingivitis and periodontitis in supragingival plaque: a cross-sectional cohort study in Malawi. Applied and Environmental Microbiology, 82(19), 6057-6067.

Skidmore, R., Kovach, R., Walker, C., Thomas, J., Bradshaw, M., Leyden, J. et al. (2003). Effects of subantimicrobial-dose doxycycline in the treatment of moderate acne. Archives of Dermatology, 139(4), 459-464.

Smiley, C.J., Tracy, S.L., Abt, E., Michalowicz, B.S., John, M.T., Gunsolley, J. et al. (2015). Systematic review and meta-analysis on the nonsurgical treatment of chronic periodontitis by means of scaling and root planing with or without adjuncts. The Journal of the American Dental Association, 146(7), 508-524.

Su, J.L., Chiou, J., Tang, C.H., Zhao, M., Tsai, C.H., Chen, P.S. et al. (2010). CYR61 regulates BMP-2dependent osteoblast differentiation through the $\alpha v \beta 3$ integrin/integrin-linked kinase/ERK pathway. Journal of Biological Chemistry, 285(41), 31325-31336.

Thummuri, D., Jeengar, M.K., Shrivastava, S., Nemani, H., Ramavat, R.N., Chaudhari, P. (2015). Thymoquinone prevents RANKL-induced osteoclastogenesis activation and osteolysis in an in vivo model of inflammation by suppressing NFKB and MAPK Signalling. Pharmacological Research, 99, 63-73.

Tomasi, C., Wennström, J.L. (2004). Locally delivered doxycycline improves the healing following nonsurgical periodontal therapy in smokers. Journal of Clinical Periodontology, 31(8), 589-595.

Tonetti, M.S., Jepsen, S., Jin, L., Otomo-Corgel, J. (2017). Impact of the global burden of periodontal diseases on health, nutrition and wellbeing of mankind: A call for global action. Journal of Clinical Periodontology, 44(5), 456-462.

Tuncer, B. B., Özmeriç, N., Tuncer, C., Teoman, İ., Çakılcı, B., Yücel, A. et al. (2005). Levels of interleukin-8 during tooth movement. The Angle Orthodontist, 75(4), 631-636.

Wiebe, C.B., Putnins, E.E. (2000). The periodontal disease classification system of the American Academy of Periodontology-an update. JournalCanadian Dental Association, 66(11), 594-599.

Wirries, A., Schubert, A.K., Zimmermann, R., Jabari, S., Ruchholtz, S., El-Najjar, N. (2013). Thymoquinone accelerates osteoblast differentiation and activates bone morphogenetic protein- 2 and ERK pathway. International Immunopharmacology, 15(2), 381386.

Yamaguchi, N., Chiba, M., Mitani, H. (2002). The induction of c-fos mRNA expression by mechanical stress in human periodontal ligament cells. Archives of Oral Biology, 47(6), 465-471.

Yimer, E.M., Tuem, K.B., Karim, A., Ur-Rehman, N., Anwar, F. (2019). Nigella sativa L. (black cumin): a promising natural remedy for wide range of illnesses. Evidence-Based Complementary and Alternative Medicine, 2019.

Yuan, H., Ma, Q., Ye, L., Piao, G. (2016). The traditional medicine and modern medicine from natural products. Molecules, 21(5), 559.

Zoch, M.L., Clemens, T.L., Riddle, R.C. (2016). New insights into the biology of osteocalcin. Bone, 82, 42-49. 